\title{
OLSR IMPROVEMENT FOR DISTRIBUTED TRAFFIC APPLICATIONS
}

\author{
Laurent Bouraoui, Arnaud de La Fortelle, Anis Laouiti \\ INRIA, Rocquencourt \\ BP 105, 78153 Le Chesnay Cedex, France \\ \{laurent.bouraoui, arnaud.de_a_fortelle, anis.laouiti\}@inria.fr
}

\begin{abstract}
This paper presents the experimental framework currently being developed at INRIA on mobile traffic applications using ad hoc communication. In this paper we propose a set of modifications to the OLSR protocol in order to adapt it to vehicle ad hoc networks. This work is the fruit of a collaboration between two INRIA research teams: HIPERCOM and IMARA. HIPERCOM is working on ad hoc routing protocols and IMARA is working on intelligent vehicles.
\end{abstract}

Keywords: ad hoc, network, OLSR, communication, traffic

\section{Wireless communication in transportation}

Mobile wireless communication has become a very popular topic to most actors in the transportation area. Car manufacturers associated with service providers want to offer new services to their customers, in order to increase comfort and safety (e.g. real time traffi c state or routing recommendations or advanced drivers assistance systems). Road authorities see the opportunity to have all embedded systems using the communication and computation power of modern vehicles to monitor and regulate the traffic avoiding investments in static equipments. Public authorities would like to increase safety and security: automatic emergency calls reduces the fatalities, vehicle transporting dangerous goods can be tracked, etc. There is a strong support for research in this area, as numerous project attest: Car2Car Communication consortium [8] or Network on Wheel [9] in Europe, the Vehicle Infrastructure Integration initiative [6] in USA or SMARTWAY [3] in Japan.

Among these applications, many of them use a centralized architecture with GPRS or UMTS data transmission. However, there are several limitations to such an architecture. 
The first limitation is the time scale: typically in the minute range. This is suffi cient for traffi $\mathrm{c}$ monitoring and control applications does not pertain to urban environments for small journeys with many different paths possibilities or local traffi c regulation (e.g. at an intersection).

The second limitation is that a Big Brother type server can not scale to the service demanded by the hundreds of thousands of cars driving in the region it covers. If all cars are communicating in real time to the server and want to adapt quickly their journey to the traffi c conditions, there will be, first, a problem of bandwidth and, second, processing difficulties, notwithstanding privacy issues.

\section{Ad hoc communication in transportation}

The possibilities and limitations of a centralized architecture draw the suitable place for distributed architecture and car ad hoc networks: for local applications that need less than a minute reaction time. There will always remain a central server who is in charge of monitoring the roads network at a regional level. But there will be local applications that run autonomously most of the time, except if there is a special need at the global level to monitor directly a given place.

In the sequel, we shortly present the OLSR protocol and the specifi c changes that are going to be implemented to fit the vehicles ad hoc networks requirements.

\section{The OLSR protocol}

$O L S R$ is an optimization of a pure link state routing protocol. It is based on the concept of multipoint relays (MPRs) [4]. First, using multipoint relays reduces the size of the control messages: rather than declaring all links, a node declares only the set of links with its neighbors that are its "multipoint relay selectors". This means that we declare only a partial set of links to the network, which is suffi cient to routing table calculation. The use of MPRs also minimizes flooding of control traffi $\mathrm{c}$. Only multipoint relays forward non duplicated control messages. In fact, each node maintains a duplicate set to prevent transmitting the same OLSR control message twice. This technique of $M P R$ signifi cantly reduces the number of retransmissions of broadcast control messages $[2,4]$. The two main $O L S R$ functionalities are, Neighbor Discovery and Topology Discovery and Dissemination. Each node in the network maintains a neighbor information database (as a result of a neighbor discovery mechanism), and a topology information database (as a result of topology discovery). These databases are refreshed periodically, and they enable each node to compute the routes to all known destinations. These routes are computed with Dijkstra's shortest path algorithm [5]. Hence, they are optimal as 
concerns the number of hops. The routing table is computed whenever there is a change in neighborhood or topology information. Further details of $O L S R$ can be found in [2].

\subsection{Using OLSR in car communication}

This article aims at adapting OLSR parameters, in order to take into account the context of car ad hoc networks. In this section, we fi rst defi ne the properties that a routing protocol should satisfy in a transportation applications. Then we introduce a set of suggested changes to the $O L S R$ protocol to meet these requirements.

3.1.1 Properties. We enumerate three properties that an ad hoc routing routing protocol should respect to be adapted to the transportation applications:

1 Enabling communication and information exchange between cars: the routing protocol should ensure and maintain a correct knowledge of the existing links and routes. Communicating car networks should be created quickly, and car appearance (i.e radio link appearance), or disappearance, should not affect, the global functioning of the network.

2 High reactivity is required: in general car traffi $\mathrm{c}$ is very dynamic. Moreover, each car can change its direction (turning left or right street for example) at any moment. The car ad hoc network should be able to react rapidly to these changes.

3 Small car networks are preferred: everyday, several thousand of cars are driving in cities at the same moment. Building an ad hoc network which include all of them is at the same time useless and infeasible. Global or distant information broadcasting is the role of a central server.

3.1.2 OLSR changes and parameters tuning. As defi ned, OLSR protocol is not well suited for wireless car communication. We now, present the modifi cations we recommend to adapt $O L S R$ to communicating transportation applications.

- The proactive behavior of $O L S R$ protocol satisfi es the fi rst property. In fact, it continuously exchanges control traffi c, by the mean of Hellos and TCs messages. As a result, all nodes can calculate routes and communicate with each other in a dynamic mobile ad hoc network. Upper layers transportation applications can simply rely on the routing information collected by $O L S R$ to have a correct view of the network topology and exchange data. 
- In order to satisfy the second property, we simply need to tune the refreshing period of topological information broacasted by $O L S R$. The default parameters values in OLSR protocol are as follow:

- Refreshing Hello period is equal to $2 \mathrm{sec}$ (i.eHELLOINTERVAL).

- Refreshing TC period is equal to $5 \sec (T C I N T E R V A L)$.

Moreover, the holding time period is usually three times the refreshing period. Therefore, a symmetric link breakage is detected after 6 seconds in worst case. This may be too long for car ad hoc networks which are moving quickly. It would be better to react on link breakage in term of one second. Hence, we suggest to reduce the HELLOJNTERVAL to some value lesser than one second. The refreshing TC period could be set to one second. In this manner, we increase the reactivity of the nodes to recover possible link breakage.

- To fulfi 11 the third requirement, we should restrict the propagation of the $O L S R$ control traffi $\mathrm{c}$ in a predefi ned area. This area should at least include the 2-hop neighbors, then nodes can select their MPRs and take profi $t$ from the optimized flooding technique of $O L S R$. The idea here is to use the geographical position which can be obtained by using Global Navigation Satellite System (GNSS, i.e. GPS or Galileo) devices embedded into the cars. This geographical information is used to drop unwanted control traffi c coming from far cars. Since the OLSR topological information are relayed by the mean of $T C$ messages, we need to include the geographical position of the originator inside these packets. For more precision, we can also add the geographical position of each address node declared in the $T C$ message. Before relaying such a message, a node have to check the distance separating it from the originator. If the distance is greater than a certain threshold, the packet will be dropped.

Each car will have a limited topology view of the global network (see fi gure 1 left). The routing table offers routes to all the nodes belonging to the predefi ned area, and the car can communicate with them directly.

Notice that the upper layer application data could be broadcasted outside these limited ad hoc networks. In fact, we can defi ne a wider area, where application data could be relayed. Of course we need to include the geographical position inside the data packet to send. In fi gure 1 right, the inner area defi nes the range for topological information relaying, and the wider area defi nes the range for application data diffusion. 

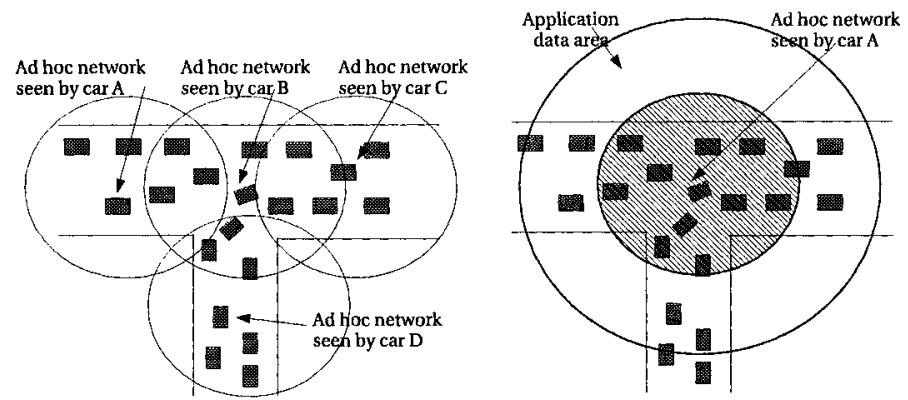

Figure 1. Ad hoc network view and data range

\section{Conclusion}

The proposed modifi cations to the $O L S R$ for vehicles ad hoc networks are currently being tested using four wheeled electric vehicles that have robotic abilities, i.e. that can be run fully autonomously: the CyCabs. We are at the beginning of the experimental phase. We are conducting range and bandwidth measurements, and testing the routing information stability in a fully mobile environment. These tests aims at tuning the different parameters of OLSR to adapt it to vhicle ad hoc networks.

\section{References}

[1] C. Adjih, T. Clausen, P. Jacquet, A. Laouiti, P. Minet, and L. Viennot. Multicast optimized link state routing. Technical Report 4721, INRIA, 2003.

[2] P. Jacquet, P. Muhletaler, P.Minet, A. Qayyum, A. Laouiti, T. Clausen, L. Viennot, and C. Adjih. Optimized link state routing protocol. In IETF RFC3626, October 2003.

[3] Japanes Ministry of Land, Infrastructure and Transport, Intelligent Transport Systems, www . its.go.jp/ITS/index/indexSmartWay . html. Smartway Project Advisory Committee.

[4] A. Qayyum, A. Laouiti, and L. Viennot. Multipoint relaying technique for fboding broadcast messages in mobile wireless networks. In HICSS: Hawai Int. Conference on System Sciences, 2002.

[5] A. S. Tanenbaum. Computer Networks. Prentice Hall, 1996.

[6] US Department of Transportation, Intelligent Transportation Systems, wwr.its.dot. gov/initiatives/initiative9. htm. Vehicle Infrastructure Integration (VII).

[7] David Ward. Developments in vehicle to vehicle communications. In Proceedings of the 9th International Forum on Advanced Microsystems for Automotive Applications, March 2005.

[8] Car2Car Communication Consortium. www. car-to-car.org.

[9] Network-on-Wheels. www.informatik.uni-mannheim.de/pi4/lib/projects/Now. 